\title{
Resting state in Alzheimer's disease: a concurrent analysis of Flash-Visual Evoked Potentials and quantitative EEG
}

\author{
Antonio Tartaglione ${ }^{1,3,6^{*}}$, Luciano Spadavecchia ${ }^{4}$, Marco Maculotti ${ }^{5}$ and Fabio Bandini ${ }^{2,3}$
}

\begin{abstract}
Background: To investigate to what extent Alzheimer's Disease (AD) affects Resting State activity, the possible impairment of independent electrophysiological parameters was determined in Eye-open and Eye-closed Conditions. Specifically, Flash-Visual Evoked Potential (F-VEP) and quantitative EEG (q-EEG) were examined to establish whether abnormalities of the former were systematically associated with changes of the latter.

Methods: Concurrently recorded F-VEP and q-EEG were comparatively analysed under Eye-open and Eye-closed Conditions in 11 Controls and 19 AD patients presenting a normal Pattern-Visual Evoked Potential (P-VEP). Between Condition differences in latencies of P2 component were matched to variations in spectral components of q-EEG.

Results: P2 latency increased in 10 AD patients with Abnormal Latency (AD-AL) under Eye-closed Condition. In these patients reduction of alpha activity joined an increased delta power so that their spectral profile equated that recorded under Eye-open Condition. On the opposite, in Controls as well as in AD patients with Normal P2 Latency (AD-NL) spectral profiles recorded under Eye-open and Eye-closed Conditions significantly differed from each other. At the baseline, under Eye-open Condition, the spectra overlapped each other in the three Groups.

Conclusion: Under Eye-closed Condition AD patients may present a significant change in both F-VEP latency and EEG rhythm modulation. The presence of concurrent changes of independent parameters suggests that the neurodegenerative process can impair a control system active in Eye-closed Condition which the electrophysiological parameters depend upon. F-VEP can be viewed as a reliable marker of such impairment.
\end{abstract}

Keywords: Alzheimer's disease, F-VEP, q-EEG, Resting state, Eye-closed, Eye-open, Dementia

\section{Background}

It has been reported that in Resting State [1], Eye-open and Eye-closed Conditions correspond to distinct patterns of activation [2-4], outlining different corticosubcortical network systems [5]. In the light of recently postulated network degeneration hypothesis $[6,7]$ it is reasonable to consider the possibility that Alzheimer's Disease (AD) affects separately either system.

Some data derived from analysis of Flash-Visual Evoked Potential (F-VEP) and quantitative-EEG (q-EEG) frequency spectra seem to support this assumption.

\footnotetext{
* Correspondence: ntntartaglione@gmail.com

'Dept of Neurology, Ospedale S. Andrea, La Spezia, Italy

${ }^{3}$ Dept of Neurology, DINOG, University of Genova, Genova, Italy

Full list of author information is available at the end of the article
}

In normal Conditions the two modalities are independent from each other and present a different behaviour in Resting State. F-VEP latency, measured at its P2 component peak, does not differ when moving from Eye-open to Eye-closed Condition [8] whereas EEG activity does, as established since Berger's first observation (1929).

$\mathrm{AD}$ may interfere with both modalities in Eye-closed Condition. As a matter of fact the increase in F-VEP latency, often reported [9-12] in $\mathrm{AD}$, has been mainly referred to this Condition [8]. By the same token, $\mathrm{AD}$ is characterized by a reduction of normal dominant posterior EEG rhythm (for reviews see [13-15]) with a significant decrease of alpha power [16,17] in Eye-closed Rest State.

Since changes in both modalities occur under the same Condition, it is possible that they reflect the involvement of a neural system active in Eye-closed Rest

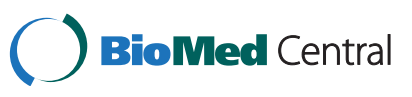


State which F-VEP and q-EEG modulations separately depend upon. If the two modalities depend on a network active in Eye-closed Condition, both should be affected by lesion of this system. Therefore, AD patients ought to present concurrent changes of F-VEP and q-EEG under Eye-closed but not under Eye-open Condition.

The data so far available are not suited to confirm such a hypothesis since F-VEP changes and EEG defects have been separately investigated in different AD studies. All underlined the individual variability of each parameter $[8,18]$. No one investigated their parallel variations in specific Conditions and in single patients. Hence it is not clear whether variations in F-VEP are associated to changes in q-EEG.

The present study was carried out to determine what is the likelihood for F-VEP changes to be associated with modifications of quantitative EEG (q-EEG) in AD patients and to ask whether the pattern of change is the same in the whole group. The result would also provide an useful criterion to identify a group of patients sharing the same functional damage and, possibly, the same distribution of damage [19].

\section{Methods}

\section{Subjects}

The study was carried out on 39 subjects, 17 Controls (10 men and 7 women) and $22 \mathrm{AD}$ patients (14 men and 8 women) consecutively admitted to the wards of the Department of Neurology of the University of Genoa. All patients agreed to participate in the study with full knowledge of the nature of the research. After a complete explanation of the study, written informed consent was obtained from each subject. The Medical Review Ethics Committee of the Department of Neuroscience, Ophthalmology and Genetics - University of Genova approved the study.

Patients affected by dementia, as defined by DSM-IV, had to meet the following criteria: a) diagnosis of Probable $\mathrm{AD}$ according to the definition of NINCDSADRDA [20]; b) degree of cooperation sufficient to carry out a Pattern-Visual Evoked Potential (P-VEP) sequence.

With a score of less than 7 at the Hachinski Ischemia Scale [21], AD patients had no evidence of focal abnormalities at CT or MR scans. Other causes - infectious, toxic, metabolic - of dementia were excluded. Controls who reported no past history of neurological or psychiatric disease were patients in the Department of Internal Medicine of the University of Genova. Information about patient's history, review of medical chart and clinical interview allowed to exclude the presence of psychiatric disturbances in Controls who underwent neurological and ophthalmological investigations to exclude the presence of specific signs or symptoms. Pupil reactivity and intraocular pressure were normal in all the patients. Visual acuity had to be no less than $15 / 20$. All the patients had normal P-VEP parameters. Cognitive changes were assessed by Mini-Mental State Examination (MMSE) [22,23] and Dementia Rating Scale (DRS) [24,25]. Patients did not differ from Controls with respect to age (t: $1.01 ; \mathrm{df}: 37$; ns) or educational level (t: 0,17 ;f: 37; ns), while they did with respect to MMSE ( $\mathrm{t}=11,7 ; \mathrm{df}: 37 ; \mathrm{P}<0.001)$ and to DRS $(\mathrm{t}=15,48$; df: 37; $\mathrm{P}<0.001)$ scores.

\section{Procedure}

q-EEG and Visual Evoked Potentials (VEPs) were recorded in a quiet room, with the subject awake, seated on a comfortable chair under continuous control. In order to avoid interference between photic stimulation and Resting State activity, F-VEP was recorded separately from EEG, in the same session.

\section{Visual evoked potentials}

VEP was recorded in 78 eyes, 34 in the Control Group and 44 in the AD one. P-VEP and F-VEP were elicited by monocular stimulation. The EEG signals, picked up with 3 silver-silver chloride electrodes placed at $\mathrm{Oz}, \mathrm{O} 1$ and $\mathrm{O} 2$ and referred to a common electrode $(\mathrm{Cz})$, were amplified at bandpass $0.53-100 \mathrm{~Hz}$ and averaged. VEPs evoked during large EEG oscillations due to head and/or ocular movements were discarded.

P-VEPs were generated by pattern reversal at $1 \mathrm{~Hz}$ frequency, using a checkerboard subtending $10^{\circ}$ with a square profile elements of $55^{\prime}$ side and a contrast of $96 \%$. No less than 256 stimuli were delivered to each eye and P100 latency was measured as the peak of the PVEP major positive component. P-VEPs were considered abnormal if their P100 latency was greater than $118 \mathrm{msec}$. and their interocular difference more than $8 \mathrm{msec}$.

F-VEP was generated at $1 \mathrm{~Hz}$ frequency by a white flash whose energy was 0.5 joules, corresponding to 10 lux of illuminance at $30 \mathrm{~cm}$ distance. The analysed epoch, i.e. $300 \mathrm{msec}$, allowed to measure the latency of P2 and N3 components of FVEP, but the study was restrained to the first component due to uncertainties in identifying the N3 peak. Peaks latencies, evaluated by two independent observers, were accepted if measures differed by less that $5 \mathrm{msec}$, otherwise components were considered "missing" and discarded. Analysis of F-VEP was done on three channels, Oz, O1, O2. Since data from $\mathrm{O} 1$ and $\mathrm{O} 2$ did not differ from those of $\mathrm{Oz}$ our report will be restricted to the latter.

Flash was presented under Eye-open and Eye-closed Conditions. The unstimulated eye was covered with an opaque patch and the patient was asked to hold his/her hand over the patched eye.

As shown in Figure 1, no less than 4 blocks of 64 trials each were collected for Eye-open and Eye-closed Conditions, in random sequence. The blocks were averaged 
A

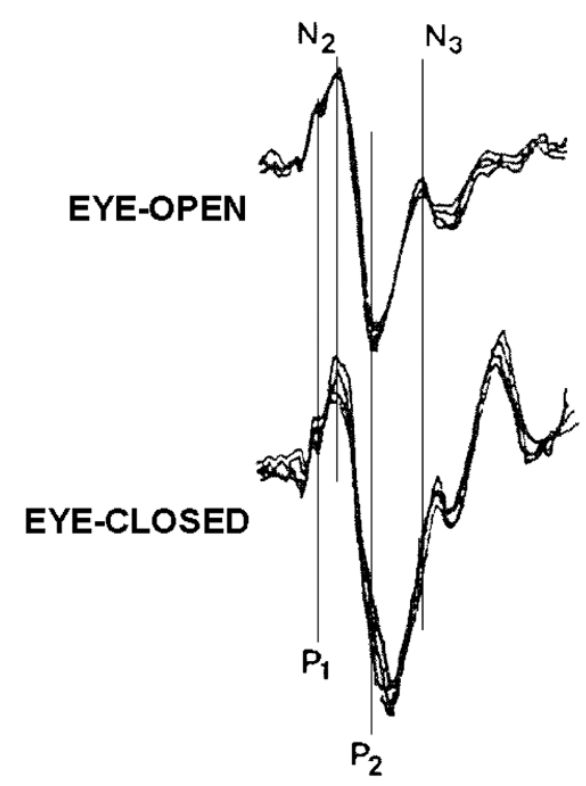

B

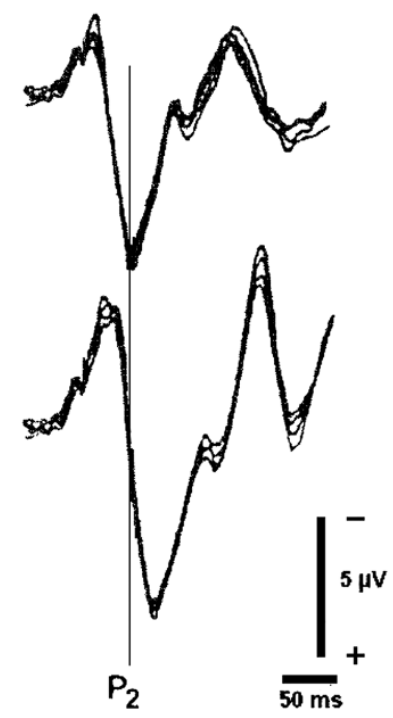

Figure 1 F-VEPs recorded in an AD-AL patient from left (A) and right (B) eye were averaged from 4 separate blocks of 64 trials. In A the distribution of components within waveform are presented. Latency of $\mathrm{P} 2$, as shown in $\mathbf{B}$, is the parameter taken into account in the study.

and latencies were measured on the final result. Each session lasted no longer than $30 \mathrm{~min}$.

9 patients presented a "missing" component on both eyes in one Condition, mainly the Eye-open one (see Results). Among the remaining 30 patients P2 latency could be identified in both eyes of all the Controls under Eye open Condition and in 9 of them under Eye-closed one (Additional file 1: Table S1). P2 latency could be identified in both eyes in 17 out of 19 AD patients under either Rest Condition. Missing values came from different patients (Additional file 1: Table S1).

Whenever present, individual P2 latencies of OD and OS significantly correlated with each other both in Eyeclosed Condition (coefficients of correlation: $r=0,85$ in Controls and $r=0,93$ in AD patients) and in Eye-open one (coefficients of correlation: $r=0,90$ in Controls and $\mathrm{r}=0,91$ in $\mathrm{AD}$ patients). Mean latencies did not differ in two eyes within group and conditions (Additional file 1: Table S1) confirming the result of correlation. This enabled us to average OD and OS latencies in order to yield a single value for each patient and condition.

\section{q-EEG}

A standard 16-lead EEG was acquired as a continuous signal for no less than 30 minutes, visually inspected for current interpretation and detection of artifacts, and stored for off-line analysis. All scalp electrodes were referenced to linked ears, and a site between Fpz and Fz was used as ground. Since the study was meant to examine mainly variations in posterior rhythms, the analysis was limited to 6 channels i.e. O1, O2, C3, C4, T3, T4. Had we extended the analysis to the whole set of channels (16) we would have found a mass of data difficult to interpret. In particular it was felt that such an extension might mask slight but significant results, due to topographical changes in different regions and in opposite directions. Electrooculogram was also recorded from tin cup electrodes placed above and below the left eye and on the outer canthus of each eye. Impedance was kept below $5 \mathrm{k} \Omega$ for all the electrodes. 10 minutes signals from the computer stored EEG were collected for each Condition, amplified, sampled by a 16 bit A/D converter with a sampling rate of $1024 \mathrm{~Hz}$. and digitally filtered in the frequency range 1$100 \mathrm{~Hz}$. No less than 27 blocks of artifact-free 2,5 s-long epochs were selected off-line by visual inspection for Eyeopen and Eye-closed Conditions.

\section{Data analysis}

Individual P2 latency was defined as the mean of the values recorded from the two eyes in Eye-open and in Eye-closed Conditions. In case of "missing" P2, the value evoked from the fellow eye was taken into account.

In order to avoid that the overlap of highly variable measures might mask possible variations among groups or between Conditions, the difference between P2 latencies recorded under Eye-open and Eye-closed Conditions was computed. The difference was considered abnormal if it outweighed the normal cut-off of $14,8 \mathrm{msec}$, i.e. the 
limit of one-tailed confidence interval in mean difference of Controls estimated with probability $p<0,001\left(t_{\alpha 0,001}\right.$, df $11=4,1)$.

Based on Rodriguez et al.'s [26] observation, q-EEG recordings obtained under either Condition were separately analysed by performing a Fast Fourier Transform (FFT) with a resolution of $0.39 \mathrm{~Hz}$ in the frequency domain between $0.39 \mathrm{~Hz}$ and $32 \mathrm{~Hz}$. The absolute values of all epoch spectrum frequencies were averaged to compute the Mean Power Spectrum (MPS) of each channel for Eye-open and Eye-closed Conditions. MPS was partitioned into 4 component bands, i.e. delta: $0.39-3.90 \mathrm{~Hz}$; theta: 4-7,8 Hz; alpha: 7,9 -12.87 Hz; beta: 12,88 - $32 \mathrm{~Hz}$. Total Power (TP) was the sum of Absolute Power (AP in $\mu \mathrm{V} 2$ ) of the four bands in each channel. AP of each component band from each channel was standardized by computing its Relative Power (RP), where RP = AP / TP, for Eye-open and Eye-closed Conditions. RP $(x)$ relative to each band was log transformed into LRP $=\log [\times /(1-x)]$ to achieve a gaussian distribution of data [27]. LRP indicates also the power of each component relative to all the others.

Overall each patient entered the study with 48 values corresponding to the following set of variables: a) 2 Conditions of recording (Eye-open and Eye-closed), b) 6 EEG channels, c) 4 component bands for each channel. A preliminary analysis was carried out in order to reduce the number of variables which was too high to be efficiently handled. Thus, individual data entered a multivariate design of analysis of variance with repeated measures [28]. Component, condition, channel, were the within subject factors and group the between-subjects one.

The condition* component"channel"group interaction was not significant, excluding the presence of systematic changes of band activities related to the different combinations of channels, conditions and groups.

On the other hand band activity averaged throughout channels showed a significant difference between conditions, apparent in all the three groups, despite the significant lower level interactions. Since our aim was that of exploring such a difference rather than describing the topographical distribution of activity on the scalp, it was decided to start from the data of single component frequency averaged throughout channels. Thus, each patient was defined by four values of AP, RP and LRP for each Condition, each value referring to a frequency component.

$\mathrm{RP}$ of individual alpha activity was compared with the corresponding sum of delta and theta RPs, by computing the Alpha /Slow Wave Ratio (A/SW) [29,30] for each Condition.

Analysis of q-EEG took into account first changes in A/SW Ratio, then variations of LRP in spectral profiles. A/SW differences among groups were analysed by
Kruskal-Wallis analysis of variance and individual comparisons by Mann-Whitney U-test. A/SW differences between Conditions within group were assessed by Wilcoxon rank test. Individual LRP data were analysed by applying a repeated measure design of ANOVA [28]. Violations of ANOVA assumptions were corrected by changing the degrees of freedom according to the Greenhouse-Geisser procedure [28]. Multiple comparisons between means were based on the Tukey's HDS test [28,31]. All data analyses were performed with PASW software (version 18.0.0).

\section{Results \\ F-VEP}

6 patients from the Control group and 3 from the AD one were excluded from further analysis, presenting a "missing" component on both eyes in one Condition or both, mainly in Eye-open one. Thus, the following data refer to 30 patients, 11 Controls ( 6 men and 5 women) and 19 AD ones (11 men and 8 women).

Table 1 reports means and SEs of latencies observed in Control and AD patients. 9 out of 19 AD patients (47\%) had a P2 latency difference lower than the normal cutoff $(14,8 \mathrm{msec})$ and they will be referred to as patients affected by AD with Normal Latency (AD-NL). 10 patients (53\%) presented a difference $\geq 14,8 \mathrm{msec}$. and they will be referred to as patients affected by AD with Abnormal Latency (AD-AL).

Group means and SEs of P2 latencies and of P2 difference between Conditions are reported in Table 2 (see also Additional file 1: Table S1). P2 latencies did not differ among groups in Eye-open Condition ( $\mathrm{F}=0,429$; df: 2, 27; $\mathrm{p}=0,66$ ). This means that the $\mathrm{AD}-\mathrm{AL}$ lengthening of $\mathrm{P} 2$ difference was due to the selective increase of P2 in Eyeclosed Condition.

\section{q-EEG}

\section{A/SW ratios}

Figure 2 presents the distribution of individual Ratios within Group and Condition. Means and SEs are reported in Table 3 (see also Additional file 1: Table S2).

Analysis of data, in Table 3 , shows that A/SW values recorded under Eye-closed Condition differed significantly from those under Eye-open one in Control group

Table 1 F-VEP - Means and standard errors of P2 latency

\begin{tabular}{lcc}
\hline & CONTROLS & AD \\
\hline EYE-OPEN (EO) & $136,7 \pm 3,6$ & $132,5 \pm 3,1$ \\
EYE-CLOSED (EC) & $140,4 \pm 3.1$ & $145,7 \pm 3,9$ \\
EC - EO & $3,7 \pm 2,6$ & $13,2 \pm 2,2$ \\
\hline
\end{tabular}

Means \pm Standard Errors of P2 latencies and of their difference between Eyeclosed (EC) and Eye-Open (EO) Conditions. Controls are compared with all the $A D$ patients. 
Table 2 F-VEP - Means and standard errors of P2 latency

\begin{tabular}{lccc}
\hline & CONTROLS & AD-NL & AD-AL \\
\hline EYE-OPEN (EO) & $136,7 \pm 3,6$ & $131,3 \pm 4,5$ & $133,5 \pm 4,6$ \\
EYE-CLOSED (EC) & $140,4 \pm 3.1$ & $137,2 \pm 4,6$ & $153,4 \pm 5,1$ \\
EC - EO & $3,7 \pm 2,6$ & $5,8 \pm 2,3$ & $19,9 \pm 1.8$ \\
\hline
\end{tabular}

Means \pm Standard Errors of P2 latencies and of their difference between Eyeclosed (EC) and in Eye-Open (EO) Conditions. AD patients are split into AD-NL and AD-AL groups.

and in $\mathrm{AD}-\mathrm{NL}$ one, while they did not in $\mathrm{AD}-\mathrm{AL}$ patients $(\mathrm{p}=0,80)$.

Kruskal-Wallis analysis of variance (Table 3) shows also that, under Eye-open, A/SW values did not differ among groups, whereas they did under Eye-closed. In this Condition all the AD-AL patients, but one, had values lower than those of Controls, the difference being significant ( $\mathrm{U}: 2 ; \mathrm{p}<0,001$, one-tailed). The $\mathrm{A} / \mathrm{SW}$ difference between $\mathrm{AD}-\mathrm{AL}$ and $\mathrm{AD}-\mathrm{NL}$ patients approached the significance ( $\mathrm{U}: 25 ; \mathrm{p}=0,06$, one-tailed), while the AD-NL group did not differ from the Control

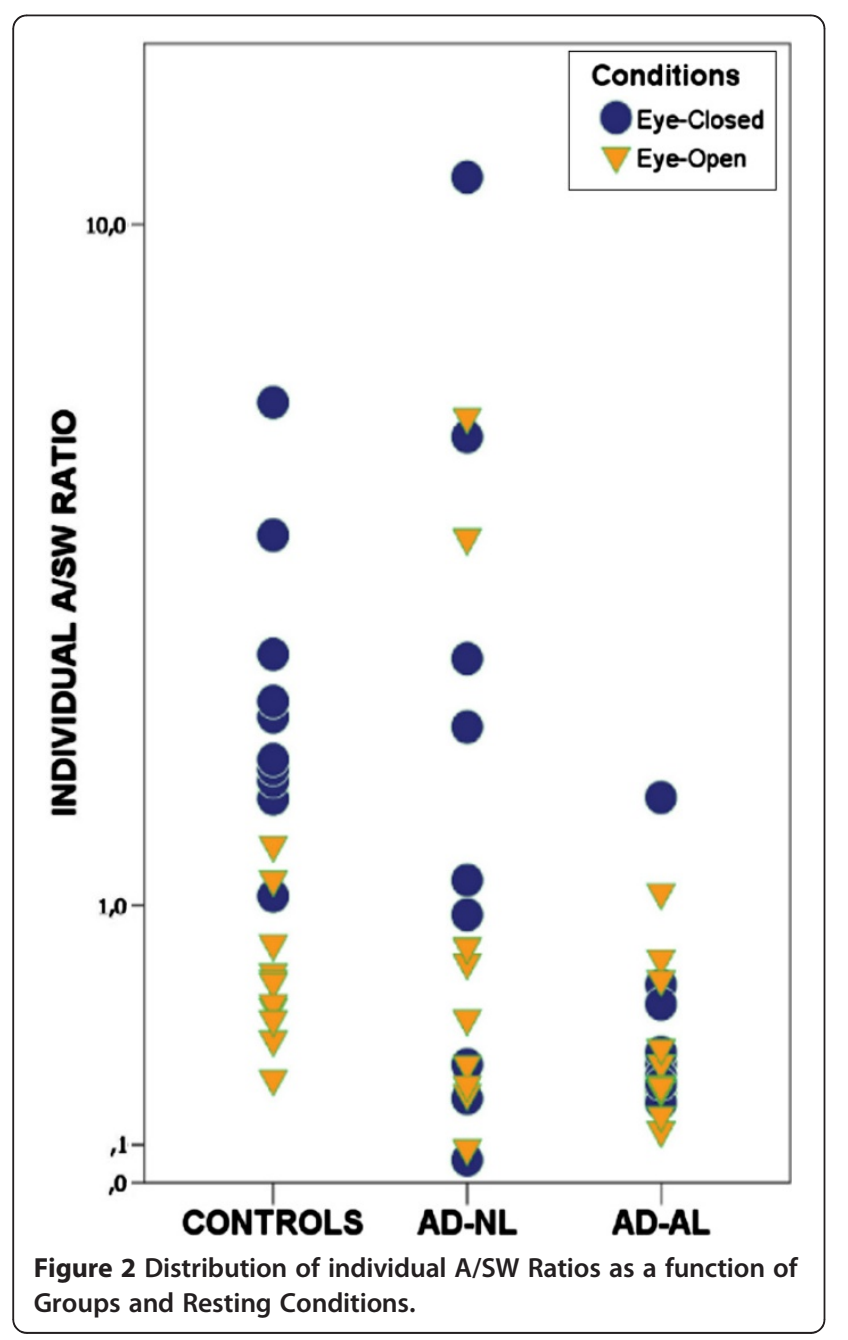

Table 3 - Means and standard errors of A /SW ratios in different groups and rest conditions

\begin{tabular}{cccc}
\hline GROUPS & EYE-OPEN & EYE-CLOSED \\
\hline CONTROL & $0,7 \pm 0,1$ & $2,5 \pm 0,4$ & Z: $-2,93 ; N=11 ; P<0,01$ \\
AD-NL & $1,4 \pm 0,7$ & $2,7 \pm 1,2$ & Z: $-2,31 ; N=9 P<0,05$ \\
AD-AL & $0,4 \pm 0,1$ & $0,5 \pm 0,1$ & Z: $-0.25 ; N=10 ; n s$ \\
& K-W: 3,$53 ;$ df: $2 ;$ ns & K-W: 12,$12 ;$ df: $2 ;$ \\
& \multicolumn{3}{c}{$P<0,01$} \\
\hline
\end{tabular}

A/SWs are averaged throughout channels. In A, Z-scores refer to the Wilcoxon rank test of A/SW Ratio difference between Conditions within Group. K-Ws refer to the Kruskal-Wallis Analysis of Variance of A/SW differences among Groups within Condition.

one (U: 36; ns). All the AD-NL patients, but three, presented A/SW values within the Controls' range.

Both the results appear clearly in Figure 2.

\section{Spectral profiles}

Table 4 presents the group means and SEs of RPs relative to the 4 component frequencies recorded under Eye-open and Eye-closed Conditions (see also Additional file 1: Table S3).

Individual LRP were entered into a design of analysis of variance with two within-subject variables, i.e. Component (alpha, beta, delta and theta frequency band), Condition (Eye-open and Eye-closed), and one between subject variable, i.e. Group (Control, AD-NL, AD-AL). The Condition* Component* Group interaction (Additional file 1: Table S4) was significant $(\mathrm{F}=4,45$; df: 15,13 ; $\mathrm{p}<0.005)$ allowing to split the analysis and to investigate each Conditions separately.

In Eye-open Condition the Component"Group interaction was not significant, (Table 5A) indicating that spectral profiles had parallel trend (Figure 3A). LRP values did not differ among groups and components. In Eye-closed Condition the Component"Group interaction (Table 5B) was significant $(\mathrm{p}=0,005)$, indicating that spectral profiles had different trends (Figure $3 \mathrm{~B}$ ) in the three groups.

Individual comparison showed that the Component"Group interaction, not significant when comparing Controls and AD-NL ( $\mathrm{F}=1,18$; df: 3,$54 ; \mathrm{p}=0,32)$, was highly significant in the contrast between Controls and AD-AL ( $F=10,73$; df: 3, 57; $p=0,001)$. In other terms the AD-NL spectral profile did not differ from that of Controls whereas the AD-AL one did (Figure $3 \mathrm{~B}$ ). In the contrast between AD-AL and AD-NL groups the Component"Group interaction approached the significance level ( $\mathrm{F}=2,1 ; \mathrm{df:} 3,51 ; \mathrm{p}=0,11)$.

Alpha band power (Figure $3 \mathrm{~B}$ ) was significantly greater in the Control Group than in AD-AL one (LRP Difference: 1,30 ; CI 95\%: 0,38/2,21; $\mathrm{p}=0.005$ ) while the opposite was true for delta component (LRP Difference: 1,20; 
Table 4 - Means and standard errors of RPs for single frequency components in different groups and rest conditions

\begin{tabular}{lccc}
\hline GROUPS & COMPONENTS & EYE-OPEN & EYE-CLOSED \\
\hline CONTROL & alpha & $24,3 \pm 2,8$ & $44,5 \pm 2,8$ \\
& beta & $36,1 \pm 3,9$ & $29,4 \pm 3,9$ \\
& delta & $21,4 \pm 2,1$ & $10,9 \pm 1,0$ \\
AD-NL & theta & $18,2 \pm 2,2$ & $15,2 \pm 2,2$ \\
& alpha & $26,0 \pm 5,2$ & $38,6 \pm 7,9$ \\
& beta & $28,1 \pm 2,6$ & $23,0 \pm 3,2$ \\
AD-AL & delta & $24,9 \pm 4,5$ & $19,8 \pm 5,6$ \\
& theta & $21,0 \pm 2,9$ & $18,6 \pm 3,8$ \\
& alpha & $19,4 \pm 3,4$ & $20,5 \pm 3,7$ \\
& beta & $29,8 \pm 3,8$ & $28,4 \pm 4,1$ \\
& delta & $26,6 \pm 3,5$ & $28,1 \pm 4,1$ \\
& theta & $24,2 \pm 3,4$ & $23,0 \pm 3,8$ \\
\hline
\end{tabular}

RPs are averaged throughout channels.

CI 95\%: $-2,09 /-0,31 ; \mathrm{p}=0.006)$. Beta and theta components did not differ in the two groups.

\section{Clinical parameters of AD patients}

Table 6 (see also Additional file 1: Table S5) presents the clinical data of Control, AD-NL and AD-AL patients. $\mathrm{AD}$ patients in the two groups did not differ from each other with respect to age, $(t=0,26 ; d f: 17$; ns), educational level ( $t=0,98$; df: 17 ; ns) and duration of the illness $(t=1,38$; df: 17 ; ns).

AD-NL patients did not differ from AD-AL both with respect to MMSE ( $\mathrm{t}=1.51$; df:17; ns) and DRS $(\mathrm{t}=1,61$; df 17; ns). The correlation coefficients between A/SW Ratios and MMSE or DRS scores were significant neither

Table 5 Analysis of Condition * Component * Group interaction

\begin{tabular}{lccccc}
\hline & SS & df & MS & F & Sig. (P) \\
\hline A. & & & & & \\
Groups ( A ) & 0,05 & 2 & 0,03 & 0,77 & 0,47 \\
Error A & 0,89 & 27 & 0,03 & & \\
Component ( B ) & 2,78 & 3 & 0,93 & 1,87 & 0,14 \\
AB & $\mathbf{3 , 4 6}$ & $\mathbf{6}$ & $\mathbf{0 , 5 8}$ & $\mathbf{1 , 1 6}$ & $\mathbf{0 , 3 4}$ \\
Error B & 40,18 & 81 & 0,5 & & \\
B. & & & & & 0,27 \\
Groups ( A ) & 0,06 & 2 & 0,03 & 1,36 & \\
Error A & 0,59 & 27 & 0,02 & & $<0,01$ \\
Component ( B ) & 16,68 & 3 & 5,56 & 7,06 & $\mathbf{0 , 0 1}$ \\
AB & $\mathbf{1 8 , 1 4}$ & $\mathbf{6}$ & $\mathbf{3 , 0 2}$ & $\mathbf{3 , 8 4}$ & \\
Error B & 63,78 & 81 & 0,79 & & \\
\hline Antys of varance
\end{tabular}

Analysis of variance of LRPs with one between (Groups) and one within (Component) variables in Eye-Open (A) and in in Eye-closed (B) Conditions. in the AD-NL group (Eye-closed: MMSE: $\mathrm{r}=0$ 0,37; DRS: $r=-0,15$ - Eye-open: MMSE: $r=0,20$; DRS: $r=-0,21$ ) nor in the AD-AL one (Eye-closed: MMSE: $r=0,09$; DRS: $r=0,33$ - Eye-open: MMSE: $r=0,24 ;$ DRS: $r=0,40$ ).

\section{Discussion}

Our analysis aimed to determine whether F-VEP modifications might be associated to q-EEG changes in $\mathrm{AD}$ patients and, in this case, whether the pattern of change were the same in the whole group.

$53 \%$ of our patients, the AD-AL ones, presented a change in F-VEP occurring selectively under Eye-closed Condition, as previously shown [8]. The remaining ones, i.e. AD-NL patients, had values within normal limits. In the first case the latency difference between Conditions was significant, in the second one it was not. Question was raised whether the delayed P2 latency might have been a mere artifact. Indeed, Coburn et al [32] maintained that, with Eye-open, F-VEP takes the form and the latencies of P-VEP. According to this hypothesis, our study would have compared the latency of P100 in Eye-open Condition with the longer one of P2 in Eye-closed one. If this were the case closure of eyes ought to have been followed by the lengthening of P2 latency in all our groups. Contrary wise, delayed P2 latency occurred only in the AD-AL group. All the others did not differ from normal.

By having ruled out the possibility of an artifact, the increased P2 latency can be considered an indirect sign of functional disorganization of brain activity in Resting State [33]. This view seems to be confirmed by two concomitant changes of q-EEG spectrum, a parameter apparently independent from F-VEP.

Firstly, the AD-AL group was characterized by A/SW values which, under Eye-closed, were significantly lower than those of Controls due to the collapse of alpha activity and the increase of delta component. The analysis of $\mathrm{AD}$-AL spectral profile confirmed the flattening of the curve where normal peaks were hardly identifiable.

Secondly, AD-AL patients did not present the normal difference between Eye-open and Eye-closed Conditions. In our Controls, indeed, alpha activity, dropped down by about $40 \%$ from Eye-closed to Eye-open state, in keeping with other observations [34], whereas in AD-AL patients the drop was less than $6 \%$. The opposite occurred for delta activity.

The flattening of the Eye-closed spectrum and the lack of difference between Eye-open and Eye-closed spectral profiles were present in 9 out of $10 \mathrm{AD}-\mathrm{AL}$ patients. This indicates that these results are consistent and robust enough to stand out immediately, despite the relative smallness of the group.

In all the $\mathrm{AD}-\mathrm{NL}$ patients, but three, $\mathrm{A} / \mathrm{SW}$ ratios were within the normal limits. The mean spectral profile recorded under Eye-closed Condition presented the same 


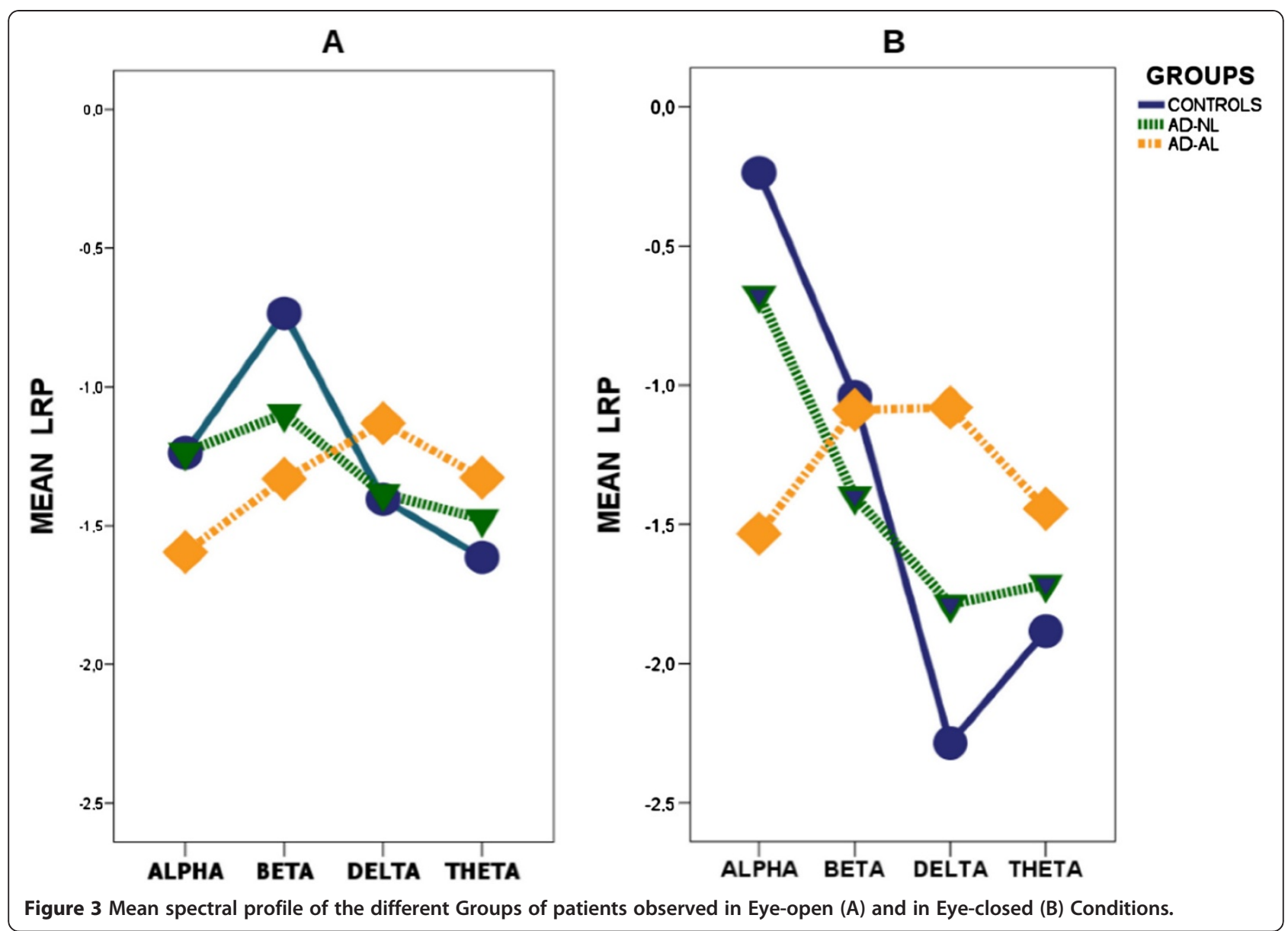

fluctuations than that of Controls and differed significantly from the one recorded under Eye-open Condition.

The association of reduced alpha and increased delta activities has been confirmed in $\mathrm{AD}$ by a wealth of observations [13-15]. Lesser attention has been paid to the changes in the spectral pattern of AD when comparing Eye-open Condition with Eye-closed one.

Table 6 Clinical data

\begin{tabular}{lccccc}
\hline & CONTROLS & & AD-NL & & AD-AL \\
\cline { 2 - 2 } & (n: 11) & & (n: 9) & & (n: 10) \\
\hline Age (years & $67,9 \pm 4,9$ & & $70,1 \pm 2,7$ & & $70,7 \pm 6,3$ \\
Schooling (years) & $6,18 \pm 1,8$ & & $5,6 \pm 3,3$ & & $7,2 \pm 3,9$ \\
Sex (N. women) & 5 & 4 & & 4 \\
Symptom duration (months) & $\#$ & & $13,2 \pm 2,4$ & & $14,7 \pm 2,2$ \\
MMSE (corrected) & $26,3 \pm 1,7$ & & $18 \pm 2,6$ & & $15,8 \pm 3,6$ \\
DRS Verbal score (raw) & $72,4 \pm 9,0$ & & $43 \pm 7,0$ & & $37,7 \pm 7,9$ \\
DRS Non-verbal score (raw) & $55,9 \pm 1,8$ & & $44,6 \pm 6,7$ & & $45,7 \pm 7,0$ \\
DRS Total score (corrected) & $0,91 \pm 0,06$ & & $0,63 \pm 0,05$ & & $0,592 \pm 0,06$ \\
\hline
\end{tabular}

Means \pm SD of clinical parameters in the 3 Groups. Patients with "missing" P2 were excluded.
Signorino et al $[16,17]$ studied the changes in 6,5-12 $\mathrm{Hz}$ band power occurring in $\mathrm{AD}$ by analysing the ratio between values observed in Eye-closed and Eye-open Conditions. Such a Reactivity Index was significantly lower than that of Controls, since the alpha activity recorded in $\mathrm{AD}$ under Eye-closed state tended to equate that of Eye-open one or to decrease below it.

Other data, derived from non-linear analysis of EEG [35], suggest that the levelling off of spectral power in Eye-closed state is not limited to the range of components explored by Signorino et al $[16,17]$ but it extends to the whole spectrum of frequencies. Pritchard et al [36] observed that in elder Controls the Global Complexity Index obtained under Eye-closed was significantly greater than under Eye-open. The difference faded out in $\mathrm{AD}$ patients where the indexes were equal. Overall, the results suggested that the $\mathrm{AD}$ process reduces the complexity of cortical dynamics underlining EEG $[13,36]$ by affecting the normal capacity to modulate brain activity in response to modified sensory information, such as after closure of eyes.

By showing the lack of difference between Eye-open and Eye-closed spectral profiles, our results confirm Pritchard 
et al.'s observation [36] but specify that changes in Resting State involve only a part, however large, of AD population.

No patient in $\mathrm{AD}$ group presented visual signs or symptoms of posterior cortical atrophy [37], nor severity of cognitive decline differed between our AD groups. Probably a difference between AD-NL and AD-AL patients, if any, should have been expected in attentional performances. As matter of fact, it is known that impairment in structures related to arousal and alertness significantly correlate with defects of attention [38] whose burden does not necessarily parallel severity of cognitive impairment [39]. The suggestion, however, needs further support as our study did not examine such an aspect.

Since F-VEP and q-EEG changes are strictly limited to Eye-closed Condition, our data support the hypothesis that behavioural states as Eye-open and Eye-closed Rest depend, at least partly, on separate system.

Such a result fits in with recent acquisitions of functional neuroanatomy which point out at different activation patterns between states [2-4,33]. In Eye-closed Rest State changing cerebral Rhythms seem to be paralleled by connectivity pattern variations $[40,42]$. Amplitude of EEG alpha rhythm, expression of thalamo-cortical and cortico-cortical synchronization under Eye closed Condition, is associated with changes in fMRI signal in occipital areas and in thalamus [4]. Correlated activities of medial thalamus and of anterior midbrain seem to precede the start of alpha activity [40].

The functional changes are due, at least partly, to neuronal activity $[3,42]$ whose pattern of activation in Eye-closed Condition involves subcortical structures mostly responsible for independent modulation of alpha rhythm and F-VEP.

The impairment of such independent parameters as FVEP and q-EEG profile under the same behavioural state, indirectly seem to support the network degeneration hypothesis according to which intrinsic connectivity networks might be the selective targets of specific neurodegenerative diseases [6,7,43]. F-VEP and Power Spectrum changes, hence, would witness the involvement of such a network system in our AD-AL patients.

In $\mathrm{AD}-\mathrm{NL}$ patients, cognitive defects were not associated with changes in the two modalities. Probably the distribution of neurodegenerative process differed from that of $\mathrm{AD}-\mathrm{AL}$ patients, though both groups presented the same level of dementia. These data suggest that the system damaged in AD-AL group has no effect on cognitive defects at least as they appear from measures drawn from Mental State Scales as MMSE or DRS. At the same time, the occurrence of contrasting results between F-VEP and q-EEG, mainly shown by those ADNL patients who presented abnormal q-EEG, confirms that the two modalities are functionally independent from each other.
Summing up, F-VEP and q-EEG changes due to AD are likely to be associated to each other under Eyeclosed Condition in a part of AD population. Such a group, identified by using F-VEP as a marker, seems to witness a change in the functional architecture of Resting State in Eye-closed Condition. Other patterns of change can occur, as suggested by the heterogeneity of AD population $[18,44]$. Whether these different patterns have distinct anatomo-clinical correlates is matter of further investigation.

\section{Additional file}

Additional file 1: Table S1. - F-VEP P2 latency (msec) as a function of group and condition. Table S2 -Individual A/SW ratios averaged throughout channels as a function of condition. Table S3 - Individual Relative Power $\left[\mathrm{RP}=(\mathrm{AP} / \mathrm{TP})^{*} 100\right]$ averaged throughout channels as a function of condition. Table S4 - Summary of main Analysis of Variance. Table S5 Clinical Data.

\section{Abbreviations}

AD: Alzheimer's Disease; AD-AL: Alzheimer's Disease with Abnormal Latency; AD-NL: Alzheimer's Disease with Normal Latency; F.VEP: Flash-Visual Evoked Potential; P-VEP: Pattern-Visual Evoked Potential; q-EEG: Quantitative-EEG; MMSE: Mini Mental State Examination; DRS: Dementia Rating Scale.

\section{Competing interests}

AT, LS, FB, MM declare no competing interests.

\section{Authors' contributors}

AT, LS, MM, FB contributed to the conception, design and execution of the research project. All the authors were involved in preparing the draft, review and critical analysis of the manuscript. AT who carried out the statistical analysis, takes responsibility for the integrity of the data and the accuracy of the data analysis and also for approval and submission of the manuscript.

\section{Acknowledgements}

The authors are indebted to prof. Emilio Favale for discussing the paper, to Maria Vittoria Gianelli for revising it and to Aldo Casaleggio - IBF - CNR - Ge for discussing non linear analysis of EEG. The support from Associazione Sistema Nervoso and from Centro di Servizi "Vivere Insieme" is gratefully acknowledged.

\section{Funding}

The study was supported by Ministero della Sanità Grant n. DGRSVE. 4-1 723 / 14.06.2001.

\section{Author details}

${ }^{1}$ Dept of Neurology, Ospedale S. Andrea, La Spezia, Italy. '2Dept of Neurology, Ospedale S. Paolo, Savona, Italy. ${ }^{3}$ Dept of Neurology, DINOG, University of Genova, Genova, Italy. ${ }^{4}$ Istituto di Biofisica, CNR Genova, Genova, Italy. ${ }^{5}$ Dept. Scienze Neuroriabilitative - Casa di Cura Privata del Policlinico s.p.a., Milano, Italy. ${ }^{6}$ Centro "La Missione" - Sarzana, La Spezia, Italy.

Received: 12 May 2012 Accepted: 15 November 2012 Published: 28 November 2012

\section{References}

1. Raichle ME, Snyder AZ: A default mode of brain function: a brief history of an evolving idea. Neuroimage 2007, 37:1083-1090.

2. Marx E, Deutschländer A, Stephan T, Dieterich M, Wiesmann M, Brandt T: Eyes open and eyes closed as rest conditions: impact on brain activation patterns. Neuroimage 2004, 21:1818-1824.

3. Bianciardi M, Fukunaga M, van Gelderen P, Horovitz SG, de Zwart JA, Duyn $\mathrm{JH}$ : Modulation of spontaneous $\mathrm{fMRI}$ activity in human visual cortex by behavioral state. Neuroimage 2009, 45:160-168. 
4. Wu L, Eichele T, Calhoun VD: Reactivity of hemodynamic responses and functional connectivity to different states of alpha synchrony: a concurrent EEG-fMRI study. Neuroimage 2010, 52:1252-1260.

5. Mesulam M: Defining neurocognitive networks in the BOLD new world of computed connectivity. Neuron 2009, 62:1-3.

6. Seeley WW, Crawford RK, Zhou J, Miller BL, Greicius MD: Neurodegenerative diseases target large-scale human brain networks. Neuron 2009, 62:42-52

7. Pievani M, de Haan W, Wu T, Seeley WW, Frisoni GB: Functional network disruption in the degenerative dementias. Lancet Neuro/ 2011, 10:829-843.

8. Tartaglione A, Bandini F, Maculotti M, Marogna M, Spadavecchia L, Favale E: Eye closure affects flash VEP latency in dementia. Electroencephalogr Clin Neurophysiol 1995, 96:197-205.

9. Visser SL, Stam FC, van Tilburg W, den Velde W, Blom JL, de Rijke W: Visual evoked response in senile and presenile dementia. Electroencephalogr Clin Neurophysiol 1976, 40:385-392.

10. Wright CE, Drasdo N, Harding GF: Pathology of the optic nerve and visual association areas. Information given by the flash and pattern visual evoked potential, and the temporal and spatial contrast sensitivity function. Brain 1987, 110:107-120.

11. Philpot MP, Amin D, Levy R: Visual evoked potentials in Alzheimer's disease: correlations with age and severity. Electroencephalogr Clin Neurophysiol 1990, 77:323-329.

12. Moore NC, Tucker KA, Jann MW, Hostetler RM, Coburn KL: Flash P2 delay in primary degenerative dementia of the Alzheimer type. Prog Neuropsychopharmacol Biol Psychiatry 1995, 19:403-410.

13. Jeong J: EEG dynamics in patients with Alzheimer's disease. Clin Neurophysiol 2004, 115:1490-1505.

14. Rossini PM, Rossi S, Babiloni C, Polich J: Clinical neurophysiology of aging brain: from normal aging to neurodegeneration. Prog Neurobiol 2007, 83:375-400

15. Rodriguez $G$, Arnaldi D, Picco A: Brain functional network in Alzheimer's disease: diagnostic markers for diagnosis and monitoring. Int J Alzheimers Dis, http://dx.doi.org /10.4061/2011/481903.

16. Signorino M, Pucci E, Belardinelli N, Nolfe G, Angeleri F: EEG spectral analysis in vascular and Alzheimer dementia. Electroencephalogr Clin Neurophysiol 1995, 94:313-325.

17. Signorino M, Brizioli E, Amadio L, Belardinelli N, Pucci E, Angeleri F: An EEG power index (eyes open vs. eyes closed) to differentiate Alzheimer's from vascular dementia and healthy ageing. Arch Gerontol Geriatr 1996, 22:245-260.

18. Jelic V, Kowalski J: Evidence-based evaluation of diagnostic accuracy of resting EEG in dementia and mild cognitive impairment. Clin EEG Neurosci 2009, 40:129-142.

19. Weintraub S, Mesulam M: With or without FUS, it is the anatomy that dictates the dementia phenotype. Brain 2009, 132:2906-2908.

20. McKhann G, Drachman D, Folstein M, Katzman R, Price D, Stadlan EM: Clinical diagnosis of Alzheimer's disease: report of the NINCDS-ADRDA Work Group under the auspices of Department of Health and Human Services Task Force on Alzheimer's Disease. Neurology 1984, 34:939-944.

21. Hachinski VC, lliff LD, Zilhka E, Du Boulay GH, McAllister VL, Marshall J, Russell RW, Symon L: Cerebral blood flow in dementia. Arch Neurol 1975, 32:632-637.

22. Folstein MF, Folstein SE, McHugh PR: Mini-mental state, a practical method for grading the cognitive state of patients for the clinician. J Psychiatr Res 1975, 12:189-198.

23. Measso G, Cavarzeran D, Zappalà G, Lebowitz B, Crook T, Pirozzolo F: The Mini-Mental State Examination: normative study of an Italian random sample. Dev Neuropsychol 1993, 9:77-85.

24. Mattis S: Mental status examination for organic mental syndromes in the elderly patients. In Geriatric psychiatry. Edited by Bellak LKT. New York: Grune and Stratton; 1976:77-121.

25. Tartaglione A, Baruzzo L, Carabelli E, De Biasi M, Guariglia G, Vivarelli F: Studio Normativo della Versione Italiana della Mattis Dementia rating scale. Giornale di Neuropsicofarmacologia 2006, 28:93-101.

26. Rodriguez G, Copello F, Vitali P, Perego G, Nobili F: EEG spectral profile to stage Alzheimer's disease. Clin Neurophysiol 1999, 110:1831-1837.

27. John ER, Ahn H, Prichep L, Trepetin M, Brown D, Kaye H: Developmental equations for the electroencephalogram. Science 1980, 210:1255-1258.

28. Winer B: Statistical principles in experimental design. 2nd edition. New York: Mc Graw-Hill; 1971.
29. Leuchter AF, Cook IA, Newton TF, Dunkin J, Walter DO, Rosenberg Thompson S, Lachenbruch PA, Weiner H: Regional differences in brain electrical activity in dementia: use of spectral power and spectral ratio measures. Electroencephalogr Clin Neurophysiol 1993, 87:385-393.

30. Gawel M, Zalewska E, Szmidt-Sałkowska E, Kowalski J: The value of quantitative EEG in differential diagnosis of Alzheimer's disease and subcortical vascular dementia. J Neurol Sci 2009, 283:127-133.

31. Daniel WW: Biostatistics: a foundation for analysis in health sciences. New York: John Wiley \& Sons; 1991.

32. Coburn KL, Amoss RT, Arruda JE, Kizer LD, Marshall YS: Effects of flash mode and intensity on P2 component latency and amplitude. Int J Psychophysiol 2005, 55:323-331.

33. Raichle ME, MacLeod AM, Snyder AZ, Powers WJ, Gusnard DA, Shulman GL: A default mode of brain function. Proc Natl Acad Sci USA 2001, 98:676-682.

34. Barry RJ, Clarke AR, Johnstone SJ, Magee CA, Rushby JA: EEG differences between eyes-closed and eyes-open resting conditions. Clin Neurophysiol 2007, 118:2765-2773.

35. Pritchard WS, Duke DW, Coburn KL, Moore NC, Tucker KA, Jann MW, Hostetler RM: EEG-based, neural-net predictive classification of Alzheimer's disease versus control subjects is augmented by non-linear EEG measures. Electroencephalogr Clin Neurophysiol 1994, 91:118-130.

36. Pritchard WS, Duke DW, Coburn KL: Altered EEG dynamical responsivity associated with normal aging and probable Alzheimer's disease. Dementia (Basel) 1991, 2:102-105.

37. Crutch SJ, Lehmann M, Schott JM, Rabinovici GD, Rossor MN, Fox NC: Posterior cortical atrophy. Lancet Neurol 2012, 11:170-178.

38. Babiloni C, Cassetta E, Binetti G, Tombini M, Del Percio C, Ferreri F, Ferri R, Frisoni G, Lanuzza B, Nobili F, Parisi L, Rodriguez G, Frigerio L, Gurzì M, Prestia A, Vernieri F, Eusebi F, Rossini PM: Resting EEG sources correlate with attentional span in mild cognitive impairment and Alzheimer's disease. Eur J Neurosci 2007, 25:3742-3757.

39. Sahakian BJ, Downes JJ, Eagger S, Evenden JL, Levy R, Philpot MP, Roberts $A C$, Robbins TW: Sparing of attentional relative to mnemonic function in a subgroup of patients with dementia of the Alzheimer type. Neuropsychologia 1990, 28:1197-1213.

40. Feige B, Scheffler K, Esposito F, Di Salle F, Hennig J, Seifritz E: Cortical and subcortical correlates of electroencephalographic alpha rhythm modulation. J Neurophysiol 2005, 93:2864-2872.

41. Yeşilyurt B, Whittingstall K, Uğurbil K, Logothetis NK, Uludağ K: Relationship of the BOLD signal with VEP for ultrashort duration visual stimuli (0.1 to $5 \mathrm{~ms}$ ) in humans. J Cereb Blood Flow Met 2010, 30:449-458.

42. Mantini D, Perrucci MG, Del Gratta C, Romani GL, Corbetta M: Electrophysiological signatures of resting state networks in the human brain. Proc Natl Acad Sci USA 2007, 104:13170-13175.

43. Brier MR, Thomas JB, Snyder AZ, Benzinger TL, Zhang D, Raichle ME, Holtzman DM, Morris JC, Ances BM: Loss of intranetwork and internetwork resting state functional connections with Alzheimer' s disease progression [abstract]. J Neurosc 2012, 32:8890.

44. Bohnen NI, Djang DSW, Herholz K, Anzai Y, Minoshima S: Effectiveness and safety of 18F-FDG PET in the evaluation of dementia: a review of the recent literature. J Nucl Med 2012, 53:59-71.

doi:10.1186/1471-2377-12-145

Cite this article as: Tartaglione et al.: Resting state in Alzheimer's disease: a concurrent analysis of Flash-Visual Evoked Potentials and quantitative EEG. BMC Neurology 2012 12:145. 